\title{
Deprescribing no Idoso
}

Ivone dos Santos Martins*

\section{RESUMO}

No doente idoso os riscos associados à polimedicação devem-se sobretudo ao aumento de interações medicamentosas, ao aumento de efeitos laterais e à má adesão terapêutica, dada a complexidade dos planos de prescrição, com agravamento subsequente das patologias de base. O deprescribing pode ser entendido como a suspensão de um fármaco, supervisionada por um médico. Devem ser ponderados para suspensão os fármacos que são responsáveis pelo aparecimento de efeitos laterais, sem qualquer indicação clínica na sua utilização, usados para combater efeitos laterais de outro fármaco entretanto já suspenso ou usados para o tratamento de uma situação clínica que entretanto já se resolveu. Existem poucos estudos sobre o melhor método de deprescribing. Contudo, a decisão de suspender determinado fármaco resulta de uma ponderação entre os objetivos terapêuticos individualizados de cada doente e da razão risco/benefício terapêutico.

Palavras-chave: Polimedicação; Idoso; Prescrição inadequada.

\section{INTRODUÇÃO}

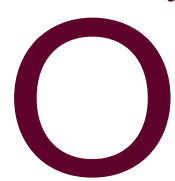

conceito não é novo, mas é inovador. O deprescribingpode ser entendido como a suspensão de um fármaco, supervisionada por um médico. ${ }^{1}$

Este conceito torna-se especialmente importante quando nos deparamos com doentes idosos polimedicados. As «cascatas de prescrição» são o resultado de um acumular de prescrições, por vezes sem qualquer benefício clínico, podendo até resultar num maior prejuízo para a saúde do doente. São diversos os fatores que podem contribuir para o somatório de prescrições: a coexistência de múltiplas morbilidades no mesmo doente, a aquisição de medicamentos não sujeitos a receita médica, a diversidade de profissionais de saúde aos quais o mesmo doente pode recorrer, entre outros. Os riscos associados à polimedicação devem-se sobretudo ao aumento de interações medicamentosas, ao aumento de efeitos laterais e à má adesão terapêutica, dada a complexidade dos planos de prescrição, com agravamento subsequente das patologias de base. ${ }^{2,3,4}$ Muitos dos fármacos utilizados no idoso são estudados em ensaios clínicos realizados em indivíduos jovens. Assim, no idoso existe ainda um risco acrescido devido à diminuição da água e da massa muscular corporal, bem como diminuição da função hepática e da função renal.

A lista mais conhecida de medicamentos inapropriados para prescrição no idoso baseia-se nos critérios

*Interna de Medicina Geral e Familiar

Unidade de Saúde Familiar Lagoa, Unidade Local de Saúde de Matosinhos de Beers. Os critérios de Beers são uma ferramenta útil que indica ao clínico quais os fármacos a evitar no idoso, quer pela sua falta de eficácia, quer pelo elevado risco de efeitos adversos. ${ }^{5}$

Mais recentemente, os critérios STOPP/START (Screening Tool of Older Person`s Prescriptions / Screening Tool to Alert doctors to Right Treatment) são também ferramentas úteis para a correta prescrição no idoso. Os critérios STOPP identificam quais os fármacos inapropriados para prescrição no idoso; cada critério é acompanhado de uma explicação concisa do motivo pelo qual o fármaco deve ser evitado na população idosa. Por outro lado, o START refere quais os fármacos que, de acordo com a patologia do doente idoso, têm evidência científica para serem prescritos com segurança. ${ }^{6}$

\section{QUANDO PONDERAR O DEPRESCRIBING?}

Recentemente têm surgido alguns ensaios clínicos que pretendem comprovar a eficácia e segurança de estratégias de deprescribing. Garfinkel et al apresenta os resultados da aplicação de um algoritmo de descontinuação terapêutica numa população de 70 doentes idosos com média de idades de 82 anos e uma média de 7,7 medicamentos por doente: $81 \%$ dos fármacos foram suspensos sem aumento significativo de efeitos adversos ao longo de 13 meses de follow-up e $88 \%$ dos doentes referiram melhoria no estado de saúde; $2 \%$ dos fármacos foram reintroduzidos devido ao reaparecimento da indicação clínica original; nenhum caso de morte foi relacionado com a suspensão do fármaco. ${ }^{7}$ 
A descontinuação de determinados fármacos deve ser ponderada em doentes polimedicados. Muitos medicamentos são prescritos à população idosa com base em estudos efetuados em populações de adultos jovens. As benzodiazepinas são, por exemplo, um dos medicamentos que devem ser evitadas no doente idoso, por maior risco de efeitos adversos e interações medicamentosas, pois podem causar sedação excessiva, aumentando o risco de quedas. ${ }^{8,9,10}$

A descontinuação de um fármaco deve também ser ponderada quando surgem efeitos laterais induzidos por este. Nos doentes idosos polimedicados, o clínico pode ter dificuldade em reconhecer uma reação adversa a determinado fármaco, podendo esta ser interpretada como uma evolução natural do processo de envelhecimento ou da patologia de base..$^{1,11}$

A ineficácia de determinado fármaco pode também constituir uma indicação para descontinuar essa terapêutica, pois não traz nenhum benefício clínico. ${ }^{1,11}$

Em doentes nos quais haja uma alteração dos objetivos terapêuticos, muitos medicamentos não têm benefício clínico acrescido: por exemplo num idoso com uma doença em fase terminal, uma terapêutica preventiva como o uso de bifosfonatos pode não ter indicação. ${ }^{1,11,12}$

Em doentes nos quais tenha havido introdução de um medicamento para tratamento de um problema de saúde entretanto resolvido deve-se ponderar a suspensão desse fármaco: por exemplo, um doente que já não necessita de tomar anti-inflamatórios não esteróides, único motivo pelo qual se introduziu um protetor gástrico, pode já não ter indicação para tomar este último. ${ }^{11,13}$

As «cascatas de prescrição» podem também ser minimizadas evitando-se a introdução de um fármaco para combater os efeitos laterais de outro fármaco: por exemplo, num doente com edema periférico por toma de antagonista de canais de cálcio, deve-se evitar introduzir um diurético para combater este edema, devendo-se antes ponderar a substituição do antagonista dos canais de cálcio por outro fármaco sobreponível em termos terapêuticos mas sem esse efeito adverso. ${ }^{13}$

\section{CONSEQUÊNCIAS DO DEPRESCRIBING}

Após a suspensão de um fármaco utilizado de forma crónica, podem advir vários resultados. A ausência de alterações do estado clínico após a suspensão do fár- maco é um dos resultados que pode ocorrer. Por outro lado, a resolução de efeitos adversos específicos confirma o efeito lateral adverso de determinado fármaco. Contudo, após a suspensão de um tratamento podem ocorrer sintomas de privação ou rebound, ou até mesmo o reaparecimento dos sintomas da doença original ou fator de risco. Nestes casos, a reintrodução da terapêutica suspensa tem indicação clínica evidente. ${ }^{1,10}$ Também se deve ter em conta que há diversos fármacos que não podem ser interrompidos de forma abrupta: por exemplo, a suspensão abrupta de um antidepressivo pode resultar no reaparecimento dos sintomas depressivos originais ou num «síndrome de descontinuação do antidepressivo", tornando difícil perceber se o reaparecimento de sintomas foi resultado da suspensão abrupta do fármaco ou do reaparecimento da doença de base. Nestes casos, o procedimento mais seguro é a redução gradual da dose do fármaco de acordo com as características específicas do fármaco em questão. ${ }^{13}$

Num estudo realizado em idosos admitidos em 6 departamentos de geriatria, ${ }^{14}$ aplicou-se um algoritmo de descontinuação terapêutica em 119 doentes; o grupo controlo incluía 71 doentes idosos sem diferenças significativas comparativamente com o grupo sujeito à descontinuação. Neste grupo foram suspensos 332 fármacos (uma média de 2,8 fármacos por doente), não se verificando efeitos adversos significativos ao longo de 12 meses de follow-up. A mortalidade foi de $21 \%$ comparativamente com $45 \%$ no grupo controlo $(p<0,001)$ e a taxa anual de referenciação a serviços de atendimento urgente foi de $11,8 \%$ comparativamente com $30 \%$ no grupo controlo $(\mathrm{p}<0,002)$.

\section{ESTRATÉGIAS DE DEPRESCRIBING}

Não existe um guia sobre como proceder ao deprescribing. Contudo, na literatura científica existem algumas linhas orientadoras que podem ajudar neste processo (figura 1).

A) O primeiro passo consiste em preparar o doente. A descontinuação de determinado fármaco implica um compromisso médico-doente e uma relação de confiança. O paciente deve ser informado da importância da toma de certos medicamentos, bem como da ausência de benefício clínico de outros, seus efeitos laterais e os riscos da 


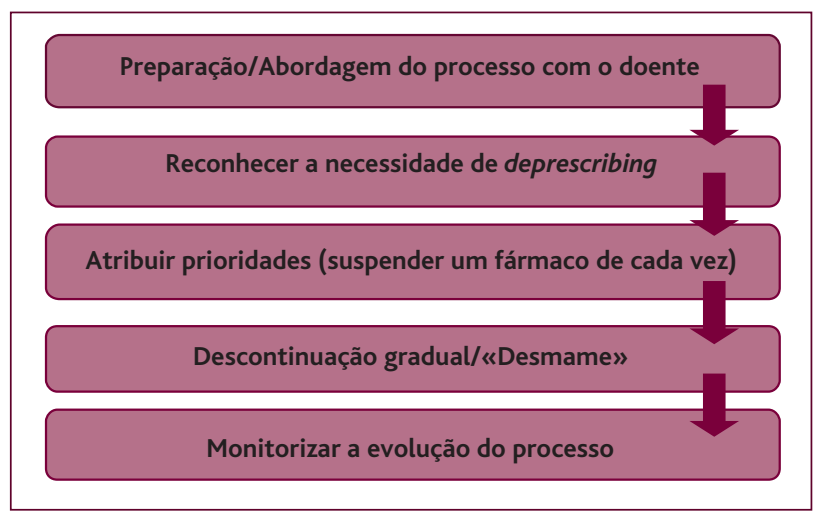

Figura 1. Abordagem geral do processo de deprescribing.

polimedicação. O sucesso do processo pode depender de uma boa comunicação médico-doente. ${ }^{1,11,13}$

B) O segundo passo consiste no reconhecimento da necessidade de deprescribing: é necessário reconhecer qual o medicamento a suspender em primeiro lugar. Assim, torna-se fundamental rever toda a medicação atual do doente: toda a medicação deve ser questionada ao doente, tendo em conta que alguma pode ser omitida, nomeadamente aquela receitada por outros médicos, prescrita numa situação aguda, produtos não sujeitos a receita médica e suplementos nutricionais. ${ }^{1,11,13}$

C) Uma vez feita a lista completa da medicação crónica, a indicação clínica de cada medicamento deve ser determinada. Isto por vezes pode implicar uma consulta a médicos de outras especialidades para determinar a adequação de determinado medicamento. Deve-se também determinar a adesão à terapêutica. Indicadores de má adesão incluem desconhecimento por parte do doente da correta posologia e horário de tomas, excesso de medicação que resta desde a última receita, medicação fora de prazo e resposta terapêutica inadequada. ${ }^{1,11,13}$

D) A identificação de reações adversas é fundamental e, por vezes, nada fácil. Isto implica um bom conhecimento dos efeitos adversos de cada medicamento, bem como possíveis interações medicamentosas, e do risco individual de cada paciente. Qualquer sintoma que tenha surgido após a introdução recente de um medicamento deve fazer suspeitar de um efeito adverso desse fármaco. ${ }^{1,11,13}$

E) Por fim deve ser possível identificar o(s) medicamento(s) a suspender e estabelecer prioridades na suspensão. Devem ser ponderados para suspensão os fármacos: que são responsáveis pelo aparecimento de efeitos laterais ou com elevado risco para desencadearem efeitos adversos, não usados correntemente, sem qualquer indicação clínica/benefício clínico na sua utilização, usados para combater efeitos laterais de outro fármaco entretanto já suspenso ou usados para o tratamento de uma situação clínica que entretanto já se resolveu. Deve-se sempre reduzir/suspender terapêuticas sequencialmente, isto é, um fármaco de cada vez, em vez de vários simultaneamente. Assim, caso se verifiquem efeitos de privação ou agravamento da doença de base, é possível identificar qual o fármaco suspenso que poderá ter de ser novamente introduzido. Também a velocidade da suspensão do fármaco deve ser individualizada: poderá haver fármacos que podem ser retirados abruptamente, já outros implicam um desmame gradual ao longo de meses. Por vezes torna-se impossível suspender terapêuticas, pelo que o objectivo deve consistir em rever o esquema geral de tratamento, de modo a simplificar o número de tomas de medicamentos ao longo do dia, maximizando desta forma a adesão terapêutica. ${ }^{1,11,13}$

F) Os pacientes devem ser regularmente vistos pelo seu médico de modo a rever o processo de $d e$ prescribing e a vigiar ao longo de todo o processo o surgimento de efeitos de privação do fármaco suspenso e/ou o agravamento das patologias de base. Não é fácil interromper tratamentos de longa data, principalmente quando o doente os considera necessários. Para o doente continuar no processo de descontinuação terapêutica é importante explicar os motivos desta suspensão, e que ele sinta os benefícios deste processo, como sentir-se melhor, gastar menos dinheiro e melhorar a sua adesão terapêutica pela simplificação do esquema terapêutico. O reforço positivo e o incentivo são muito importantes. ${ }^{1,11,13}$ 


\section{CONCLUSÃO}

Mais importante que interromper terapêuticas é a revisão de todo o plano terapêutico do doente, de modo a simplificar o seu esquema de tratamento, aumentar a sua adesão e desta forma maximizar o benefício clínico dos tratamentos instituídos. Os clínicos são muitas vezes relutantes em interromper terapêuticas, especialmente se não foram eles que as iniciaram. É consensual que alguns fármacos sujeitam os doentes idosos a riscos e efeitos adversos graves com benefício clínico muito limitado. Existem poucos estudos sobre o melhor método de deprescribing e muito poucos ensaios clínicos que suportem uma metodologia concreta neste processo. Contudo, a decisão de suspender determinado fármaco resulta de uma ponderação entre os objetivos terapêuticos individualizados de cada doente e da razão risco/benefício terapêutico.

\section{REFERÊNCIAS BIBLIOGRÁFICAS}

1. Le Couteur D, Banks E, Gnjidic D, McLachlan A. Deprescribing. Aust Prescr 2011, 34 (6): 182-5.

2. Hilmer $S N$, Gnjidic D. The effects of polypharmacy in older adults. Clin Pharmacol Ther 2009 Jan; 85 (1): 86-8.

3. Chan $M$, Nicklason F, Vial JH. Adverse drug events as a cause of hospital admission in the elderly. Intern Med J 2001 May-Jun; 31 (4): 199205.

4. Hamilton HJ, Gallagher PF, O'Mahony D. Inappropriate prescribing and adverse drug events in older people. BMC Geriatr 2009 Jan 28; 9: 5.

5. American Geriatrics Society 2012 Updated Beers Criteria for Potentially Inappropriate Medication Use in Older Adults. Disponível em: http://www.americangeriatrics.org/health_care_professionals/clinical_practice/clinical_guidelines_recommendations/2012 [acedido em 29/10/2012].

6. Gallagher P, Ryan C, Byrne S, Kennedy J, O'Mahony D. STOPP (Screening Tool of Older Person's Prescriptions) and START (Screening Tool to
Alert doctors to Right Treatment): Consensus Validation. Int J Clin Pharmacol Ther 2008 Feb; 46 (2): 72-83.

7. Garfinkel D, Mangin D. Feasibility study of a systematic approach for discontinuation of multiple medications in older adults: addressing polypharmacy. Arch Intern Med 2010 Oct 11; 170 (18): 1648-54.

8. Hardy J, Hilmer S. Deprescribing in the last year of life. J Pharm Pract Res 2011 Jun; 41 (2): 146-51.

9. Boyle N, Naganathan V, Cumming RG. Medication and falls: risk and optimization. Clin Geriatr Med 2010 Nov; 26 (4): 583-605.

10. Campbell AJ, Robertson MC, Gardner MM, Norton RN, Buchner DM. Psychotropic medication withdrawal and a home-based exercise program to prevent falls: a randomized, controlled trial. J Am Geriatric Soc 1999 Jul; 47 (7): 850-3.

11. Woodward M. Deprescribing: Achieving better health outcomes for older people through reducing medications. J Pharm Pract Res 2003 Dec; 33 (4): 323-8.

12. Holmes HM. Rational prescribing for patients with reduced life expectancy. Clin Pharmacol Ther 2009 Jan; 85 (1): 103-7.

13. A practical guide to Stopping Medicines in Older People. Best Pract J 2010; 27: 10-23. Disponível em: http://www.bpac.org.nz/magazine/2010/april/stopGuide.asp [acedido em 29/10/2012].

14. Garfinkel D, Zur-Gil S, Ben-Israel J.The war against polypharmacy: a new cost-effective Geriatric-Palliative Approach for improving drug therapy in disabled elderly people. Isr Med Assoc J 2007 Jun; 9 (6): 430-4.

\section{CONFLITO DE INTERESSES}

A autora declara não existirem conflitos de interesses relacionados com o conteúdo do artigo.

\section{ENDEREÇO PARA CORRESPONDÊNCIA}

Ivone dos Santos Martins

Rua da Lagoa, 4460 Senhora da Hora, Matosinhos

Email: isantosmts@gmail.com

Recebido em 31/10/2012

Aceite para publicação em 19/01/2013

Artigo escrito ao abrigo do novo acordo ortográfico.

\section{ABSTRACT}

\section{DEPRESCRIBING IN THE ELDERLY}

In elderly patients, the risks associated with polypharmacy are due to drug interactions, adverse effects, and poor compliance with complex treatment plans, leading to worsening of underlying conditions. Deprescribing is the cessation of therapy supervised by a clinician. Drugs that should be considered for cessation are those responsible for side effects, those not clinically indicated, drugs used to treat side effects of another drug that has already been stopped, or drugs used for the treatment of a situation that has resolved. There are few studies of the best method of deprescribing. The decision to stop a drug arises from the balance between therapeutic goals and the risk/benefit ratio of treatment.

Key words: Polypharmacy; Aged; Inappropriate Prescribing. 